\title{
SUPLEMENTAÇÃO ALIMENTAR DE CABRAS ANGLO-NUBIANAS NA ÉPOCA CHUVOSA, NA REGIÃO SEMI-ÁRIDA DO NORDESTE BRASILEIRO'
}

\author{
NELSON NOGUEIRA BARROS ${ }^{2}$, ROBERTO CÉSAR MAGALHÃES MESQUITA ${ }^{3}$, \\ MARCELO RENATO ALVES DEARAÚJO ${ }^{3}$ e RUBÊNIO BORGES DE CARVALHO ${ }^{3}$
}

\begin{abstract}
RESUMO - Foi realizado um experimento para avaliar a influência da suplementação alimentar na produção de leite de cabras da raça Anglo-nubiana, durante a época chuvosa, na região semi-árida do Nordeste brasileiro. Vinte e quatro cabras foram mantidas em pastagem nativa rebaixada, distribuídas ao acaso, por tipo de parto e produção de leite, em três níveis de suplementação: $\mathrm{N}_{0}$ - sem suplementação, $\mathrm{N}_{1}-150 \mathrm{~g}$ de concentrado/animal/dia e $\mathrm{N}_{2}-300 \mathrm{~g}$ de concentrado/animal/dia. Não houve diferença significativa $(\mathrm{p}>0,05)$ entre os níveis de suplementação. A produção de leite foi descrita por uma regressão múltipla em função do período de lactação em semana. A interação semana x níveis de suplementação foi significativa $(\mathrm{p}<0,01)$. Os resultados revelam que a suplementação com concentrado, na dose de $300 \mathrm{~g} / \mathrm{animal} / \mathrm{dia}$, foi a melhor opção para se produzir leite com cabras Anglo-nubianas, durante a época chuvosa
\end{abstract}

Termos para indexação: caprino, produção de leite, caatinga nativa melhorada.

\section{FOOD SUPPLEMENTATION OF ANGLO-NUBIAN GOATS IN RAINY SEASON IN SEMI-ARID REGION OF NORTHEAST BRAZIL}

\begin{abstract}
This experiment was carried out to evaluate the influence of food supplementation on milk production of Anglo-nubian goats, during the rainy season, in semi-arid region of Northeast Brazil. Twenty four goats were maintained in lowered "caatinga", distributed randomly according to the parturition order and milk production in three supplementation levels: $\mathrm{N}_{0}$ - no supplementation, $\mathrm{N}_{1}$ $150 \mathrm{~g}$ of concentrate/animal/day and $\mathrm{N}_{2}-300 \mathrm{~g}$ of concentrate/animal/day. There was no significant difference $(\mathrm{p}>0.05)$ between supplementation levels. Milk production was described by multiple regression to lactation period, in week. The results showed that the best option to produce milk with Anglo-nubian, during the wet season, is $300 \mathrm{~g}$ of concentrate/animal/day.
\end{abstract}

Index terms: goat, milk production, lowered caatinga.

\section{INTRODUÇ̃̃O}

A caprinocultura leiteira, no Brasil, vem crescendo substancialmente nos últimos anos. Este crescimento é refletido no aumento da produção de leite caprino nacional, no período de 1982 a 1992 (52\%), ao passo que o mundial foi de apenas 37\% (Quarterly Bulletin of Statistics 1988, 1993). Vários fatores têm contribuído para este crescimento, dentre os quais se destacam: a fácil adaptação do caprino aos diversos ecossistemas (French, 1970;

\footnotetext{
1 Aceito para publicação em 2 de março de 1999.

2 Méd. Vet., M.Sc., Embrapa-Centro Nacional de Pesquisa de Caprinos (CNPC), Caixa Postal D-10, CEP 62011-970 Sobral, CE. E-mail: nelson@cnpc.embrapa.br

${ }^{3}$ Eng. Agr., M.Sc., Embrapa-CNPC.
}

Devendra, 1980; Gutierrez \& De Boer, 1982), as boas cotações que o leite alcança no mercado, e a existência de demanda por produtos derivados do leite da cabra (Neumaier, 1986).

A região semi-árida do Nordeste brasileiro caracteriza-se por apresentar uma época chuvosa que ocorre durante os meses de janeiro a junho, e outra, seca, de julho a dezembro. Durante a época chuvosa, a fitomassa pastável é abundante e de boa qualidade nutricional. Todavia, durante a época seca, além da queda acentuada na disponibilidade da fitomassa pastável, há acentuado aumento da fração fibrosa aliado a um rápido processo de lignificação da parede celular das plantas forrageiras. Estes efeitos, agindo conjuntamente, reduzem o consumo de nutrientes e o desempenho animal. Pesquisas desenvolvidas no município de 
Sobral (Pfister, 1983; Kirmse, 1984; Schacht et al., 1992) mostraram que proteína e, principalmente, energia, são deficientes para crescimento de pequenos ruminantes durante a época seca. Barros et al. (1992) mostraram que o aumento do nível de energia na dieta de cabras Anglo-nubiana, em pastejo no semi-árido, durante a época chuvosa, não influenciou a produção de leite, porém os autores não testaram o nível zero de suplementação.

O estudo teve a finalidade de verificar o efeito da suplementação alimentar em cabras de raça Anglonubiana em pastejo, durante a época chuvosa, no semi-árido.

\section{MATERIAL E MÉTODOS}

A pesquisa foi conduzida na Embrapa-Centro Nacional de Pesquisa de Caprinos (CNPC), situada no norte do Estado do Ceará, região semi-árida do Nordeste do Brasil. A precipitação média da região é de $722 \mathrm{~mm} / \mathrm{ano}$, e a temperatura é elevada, com pequenas variações ao longo do ano. As médias anuais da máxima e da mínima são de $35^{\circ} \mathrm{C}$ e $22^{\circ} \mathrm{C}$, respectivamente.

Foram utilizadas 24 cabras da raça Anglo-nubiana de segunda e terceira ordem de parto, pesando, em média, $40,6 \pm 0,8 \mathrm{~kg}$ de peso vivo

O estudo teve início em 5/5/1988, com duração de 13 semanas, e a coleta de dados foi realizada entre a segunda e a décima quarta semana de lactação. Os animais foram mantidos em pastagem nativa raleada, das 8 às $16 \mathrm{~h}$, numa lotação de 1,0 animal/ha/ano. A suplementação alimentar foi feita com concentrado (Tabela 1 ), contendo $15,4 \%$ de proteína bruta, 3,34 Mcal de energia digestível $/ \mathrm{kg}$ de matéria seca, $0,44 \%$ de cálcio e $0,48 \%$ de fósforo, nos seguintes níveis: $\mathrm{N}_{0}$, sem suplementação; $\mathrm{N}_{1}, 150 \mathrm{~g}$ de concentrado/ animal/dia, e $\mathrm{N}_{2}, 300 \mathrm{~g}$ de concentrado/animal/dia. A ração foi oferecida, individualmente, no período da tarde, após os animais retornarem ao aprisco, e o consumo de matéria seca da ração foi medido diariamente. Água e sal mineral estiveram à disposição dos animais no aprisco.

Os cabritos foram separados das respectivas mães sete dias após o nascimento, e aleitados artificialmente. As cabras foram ordenhadas diariamente, às $7 \mathrm{e}$ às $16 \mathrm{~h}$ e o controle leiteiro efetuado em três dias da semana. Na sexta semana de experimento, foram coletadas, individualmente, amostras de leite, para análise de gordura (método de Babcok), proteína bruta e sólidos totais (Association of Official Agricultural Chemists, 1975). Os animais foram pesados ao parto, ao início do experimento, e, em seguida, a cada sete dias.
O delineamento experimental foi o inteiramente casualizado, com oito animais por tratamento, perfazendo um total de 24 cabras. Como o período experimental foi de 13 semanas, com as lactações mensuradas durante três dias em cada semana (S), a produção de leite foi analisada pelo seguinte modelo estatístico, o qual caracteriza o splitplot no tempo

$\mathrm{Y}=\mu+\mathrm{N}_{\mathrm{i}}+\mathrm{A}_{\mathrm{j}}\left(\mathrm{N}_{\mathrm{i}}\right)+\mathrm{S}_{\mathrm{k}}+(\mathrm{NS})_{\mathrm{ik}}+\mathrm{E}_{\mathrm{ijk}}$

onde:

$\mu=$ média geral;

$\mathrm{N}_{\mathrm{i}}=$ efeito dos níveis de suplementação;

$\mathrm{A}_{\mathrm{j}}\left(\mathrm{N}_{\mathrm{i}}\right)=$ efeito dos animais dentro de cada nível de suplementação, usado como erro para medir o efeito de cada um destes níveis;

$\mathrm{S}_{\mathrm{k}}=$ efeito da semana;

$(\mathrm{NS})_{\mathrm{ik}}=$ interação entre níveis de suplementação e semanas;

$\mathrm{E}_{\mathrm{ijk}}=$ erro experimental.

Como os efeitos dos níveis de suplementação são de natureza quantitativa, a aplicação de testes de compensação de médias para estes efeitos não é correta. Assim, depois de aplicado o teste $\mathrm{F}$, o comportamento dos diferentes níveis de suplementação foi observado mediante a análise de regressão.

Finalmente, procedeu-se a uma análise econômica dos níveis de suplementação, por meio do cálculo da margem bruta resultante da produção de leite. De acordo com Santos et al. (1997), a margem bruta consiste na diferença entre a receita bruta - produção $\mathrm{x}$ preço de venda no mercado - e os custos variáveis - custos com concentrado, forragem, vacinas, vermífugo e mão-de-obra.

O uso de mão-de-obra para o arraçoamento dos animais e a limpeza das instalações foi estimado em 1,5 hora/homem/dia para o nível sem concentrado $\left(\mathrm{N}_{0}\right)$ e 2,0 hora/homem/dia para cada nível com concentrado $\left(\mathrm{N}_{1} \mathrm{e}\right.$ $\mathrm{N}_{2}$ ). Assim, o custo deste item foi estimado com base no salário mínimo vigente $(\mathrm{R} \$ 130,00)$, acrescido de $40 \%$ de encargos sociais. O custo da forragem foi estimado com base no consumo de matéria seca, correspondente a $4 \%$ do peso vivo do animal, no custo de implantação da pastagem, e no rendimento de forragem. O preço do leite foi considerado a $\mathrm{R} \$ 0,80$ por litro, correspondendo ao valor comercializado atualmente no mercado nordestino.

As margens brutas encontradas foram submetidas a análise de variância, a 5\% de probabilidade, para identificar diferença significativa entre níveis de suplementação. 


\section{RESULTADOS E DISCUSSÃO}

Na Tabela 2, é mostrada a análise de variância da produção de leite. O coeficiente de variação do experimento foi de $12,24 \%$. O coeficiente de determinação $\left(r^{2}=0,86\right)$ demonstra que o modelo utilizado é adequado.

As produções de leite obtidas em função dos níveis de suplementação e dos períodos de lactação (semana) estão apresentadas na Tabela 3 . O efeito de nível de suplementação não influenciou $(\mathrm{p}>0,05)$ a produção de leite (Tabela 2). Os resultados mostraram que somente a caatinga nativa rebaixada $\left(\mathrm{N}_{0}\right)$ suportou uma produção de leite de $1,2 \mathrm{~kg} / \mathrm{dia}$ (Tabela 3). Os incrementos nos níveis $\mathrm{N}_{1}$ e $\mathrm{N}_{2}$ em relação ao $\mathrm{N}_{0}$, embora não-significativos, foram de $8 \%$ e $23 \%$, respectivamente (Fig. 1). Isto indica que a pastagem era de boa qualidade nutricional, porém insuficiente para atender às necessidades nutricionais dos animais em níveis de produção mais elevados. A precipitação ocorrida no ano do experimento foi inferior à média dos anos de 1988 a 1995

TABELA 1. Composição da ração utilizada na suplementação das cabras ${ }^{1}$.

\begin{tabular}{lcc}
\hline Itens & $\begin{array}{c}\text { Matéria natural } \\
(\%)\end{array}$ & $\begin{array}{c}\text { Matéria seca } \\
(\%)\end{array}$ \\
\hline Farelo de soja & 16,8 & 15,12 \\
Milho em grãos & 81,0 & 72,09 \\
Suplemento mineral & 1,2 & 1,2 \\
Sal comum & 1,0 & 1,0 \\
\hline
\end{tabular}

1 Composição, por kg do produto: cálcio, $240 \mathrm{~g}$; fósforo, $174,8 \mathrm{~g}$; enxofre $24 \mathrm{~g}$; magnésio, $10 \mathrm{~g}$; cobre, $800 \mathrm{mg}$; cobalto, $50 \mathrm{mg}$; zinco, $4.000 \mathrm{mg}$ ferro, $3.100 \mathrm{mg}$; manganês, $2.000 \mathrm{mg}$; iodo, $80 \mathrm{mg}$; fluor, $1,7 \mathrm{mg}$ (máximo)

TABELA 2. Análise da variância da produção de leite de cabras de raça anglo-nubiana submetidas a três diferentes níveis de suplementação alimentar, em pastoreio na região semi-árida.

\begin{tabular}{lcrrrc}
\hline Fonte de variação & $\begin{array}{c}\text { Grau de } \\
\text { liberdade }\end{array}$ & $\begin{array}{c}\text { Soma dos } \\
\text { quadrados }\end{array}$ & \multicolumn{1}{c}{$\begin{array}{c}\text { Quadrado } \\
\text { médio }\end{array}$} & F & $\begin{array}{c}\text { Nível de } \\
\text { significância }\end{array}$ \\
\hline Nível de suplementação (NS) & 2 & 251471516,9 & 125735758,4 & 2,95 & 0,0734 \\
Animal (tratamento) & 22 & 938559969,9 & 42661816,8 & 32,11 & 0,0001 \\
Semana (S) & 12 & 901015860,6 & 75084655,1 & 56,52 & 0,0001 \\
NS x S & 24 & 59391689,3 & 24744652,5 & 1,86 & 0,0100 \\
Erro & 264 & 300744581,0 & 1328578,0 & & \\
\hline
\end{tabular}

(Fig. 2), porém suficiente para promover a produção de fitomassa pastável, o que explica a inexistência de diferença significativa entre níveis de suplementação. Barros et al. (1992) não observaram diferença significativa $(p>0,05)$ na produção de leite de cabras Anglo-nubianas submetidas a suplementação energética em condições de pastejo semelhantes ao deste estudo. As produções de leite obtidas foram: $1,4 \mathrm{~kg}, 1,4 \mathrm{~kg}, 1,4 \mathrm{~kg}$ e $1,3 \mathrm{~kg}$ de leite/ animal/dia para os níveis de suplementação energética de $0,8,1,6,2,4$ e 3,2 Mcal de energia digestível/animal/dia, respectivamente.

Observando-se a curva de produção de leite (Fig. 1), nota-se que os animais submetidos ao nível de suplementação $\mathrm{N}_{1}$ não apresentaram pico de lactação, que geralmente ocorre entre a terceira e a quarta semana de produção de leite. Este comportamento foi inesperado e inexplicável. Barros et al. (1992) trabalhando com cabras da raça Anglonubiana, em condições similares à deste experimento, mostraram que no início da lactação houve aumentos na produção de leite, e que o valor máximo (pico) ocorreu na quarta semana de lactação. Pela Fig. 3, observa-se perda de peso dos animais até o pico da lactação (terceira semana do experimento, quarta de lactação). A partir daí, os animais passaram a recuperar o peso, e chegaram ao final do estudo com o peso ligeiramente superior ao verificado no início. Estes resultados estão em conformidade com o National Research Council (1981), que prevê, nas tabelas de requerimentos nutricionais de caprinos leiteiros, uma perda de peso de cerca de $20 \mathrm{~g} /$ dia para cabras ao início da lactação.

Sands \& McDowell (1978) observaram que a produção média diária de leite de cabras da raça Anglonubiana em áreas tropicais e subtropicais foi de 
$0,89 \mathrm{~kg} / \mathrm{animal} / \mathrm{dia}$ em um período de 230 dias, enquanto a média dos Estados da Paraíba, Pernambuco e Rio Grande do Norte (Baker \& Souza Neto, 1989) foi de $0,80 \mathrm{~kg} / \mathrm{animal} / \mathrm{dia}$ em 134 dias de lactação. Também, Zometa et al. (1986) obtiveram produções médias diárias de leite em cabras da raça Anglonubiana, alimentadas com rações contendo ou não uréia, em confinamento por 23 semanas, inferiores às observadas neste estudo. Rodrigues et al. (1992),

TABELA 3. Valores médios de produção de leite de cabras de raça Anglo-nubiana submetidas a três níveis de suplementação alimentar, em pastoreio na região semi-árida.

\begin{tabular}{cc}
\hline Item & $\begin{array}{c}\text { Produção de leite } \\
\text { (kg/animal/dia) })^{1}\end{array}$ \\
\hline Nível de suplementação & 1,21 \\
$\mathrm{~N}_{0}$ & 1,30 \\
$\mathrm{~N}_{1}$ & 1,50 \\
$\mathrm{~N}_{2}$ & \\
Semana & 1,57 \\
1 & 1,63 \\
2 & 1,64 \\
3 & 1,61 \\
4 & 1,57 \\
5 & 1,49 \\
6 & 1,36 \\
7 & 1,26 \\
8 & 1,16 \\
9 & 1,12 \\
10 & 1,09 \\
11 & 1,01 \\
12 & 1,00 \\
13 & 12,24 \\
$\mathrm{CV}(\%)$ & 0,86 \\
$\mathrm{r}^{2}$ & \\
\end{tabular}

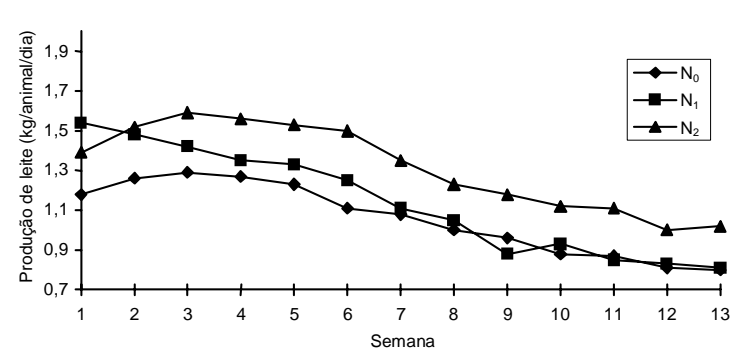

FIG. 1. Produção de leite das cabras durante o período experimental.

Pesq. agropec. bras., Brasília, v.34, n.11, p.2151-2156, nov. 1999 trabalhando com cabras da raça Anglonubiana em pastejo de capim-bufel (Cenchrus ciliaris) e suplementadas com $800 \mathrm{~g}$ de concentrado/animal/dia, nas 16 primeiras semanas de lactação, obtiveram uma produção de leite de $1,22 \mathrm{~kg} / \mathrm{animal} / \mathrm{dia}$, equivalente aos níveis de suplementação $\mathrm{N}_{1}$ e $\mathrm{N}_{2}$ obtidos no presente trabalho. Isto sugere que a caatinga rebaixada tem maior potencial para produção de leite com cabras que o capim-bufel, pois os animais sem suplementação $\left(\mathrm{N}_{0}\right)$ ou suplementados com $300 \mathrm{~g} / \mathrm{dia}\left(\mathrm{N}_{1}\right)$ de concentrado, apresentaram produções de leite semelhantes àquelas em pastejo de capim-bufel e suplementadas com $800 \mathrm{~g} /$ dia de concentrado.

A produção de leite $(\mathrm{Y})$ foi descrita por uma regressão múltipla em função do período de lactação em semana (S). Na Tabela 4, encontra-se a estimativa dos parâmetros da equação estrutural. O modelo ajustado explica as variações na produção

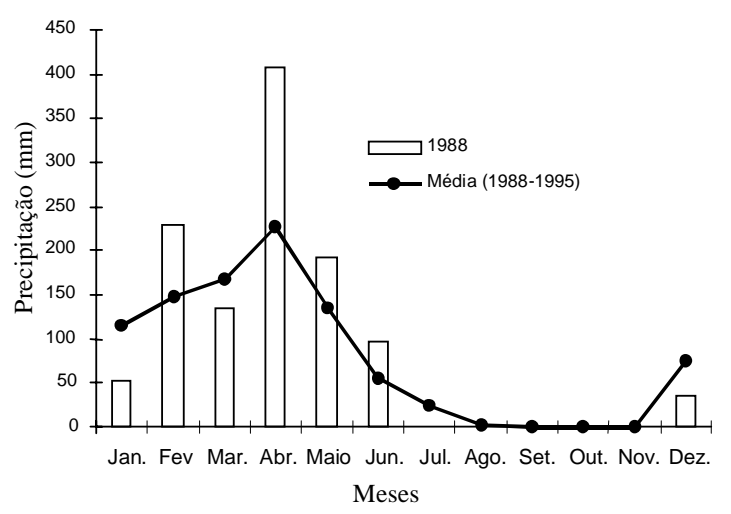

FIG. 2. Precipitação no local do experimento nos anos de 1988 a 1995.

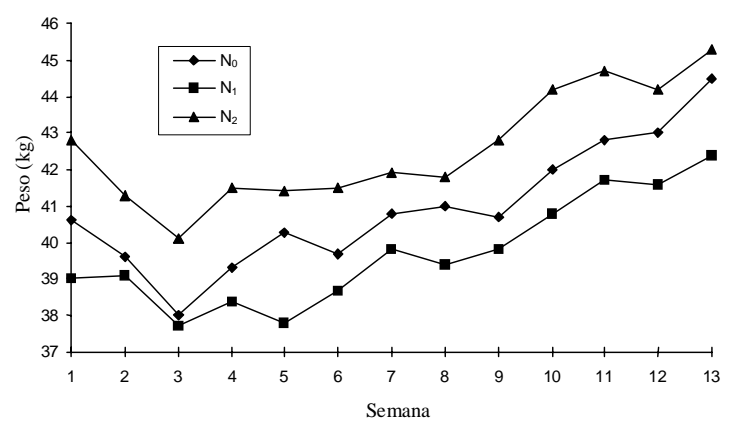

FIG. 3. Peso corporal das cabras durante o período experimental. 
de leite em função do período de lactação, referendado pelas estatísticas que acompanham a regressão. O sinal negativo indica o declínio na produção logo após o pico da lactação, o que mostra que a persistência do pico de lactação foi restrita, mesmo que as condições alimentares tenham sido satisfatórias, à semelhança do que foi constatado por Barros et al. (1992).

Na Tabela 5, estão apresentados os percentuais de proteína bruta, sólidos totais e gordura do leite. Os resultados obtidos neste estudo assemelham-se aos observados por Barros et al. (1992), no tocante à proteína do leite, mas foram inferiores aos verificados por estes autores, no que se refere aos teores de gordura e de sólidos totais. A proteína bruta do leite não foi influenciada pelos níveis de suplementação. Todavia, observando-se os dados relativos à gordura, visualiza-se uma tendência de redução na gordura do leite, na medida em que se elevou o nível de

TABELA 4. Estimativa dos coeficientes da equação de regressão da produção de leite de cabras da raça Anglo-nubiana em função do período de lactação (semana, S), na região semi-árida ${ }^{1}$.

\begin{tabular}{cccccc}
\hline $\begin{array}{c}\text { Constante } \\
\text { da regressão }\end{array}$ & \multicolumn{5}{c}{ Variáveis explicativas $^{2}$} \\
\cline { 2 - 6 } & $\mathrm{S}$ & $\mathrm{S}^{2}$ & $\mathrm{~S}^{3}$ & $\begin{array}{c}\mathrm{X}^{2} \\
\text { (ajustado) }\end{array}$ & $\mathrm{F}$ \\
\hline $10,272^{* *}$ & $0,957^{* * *}$ & $-0,217 *$ & $0,009 *$ & 0,99 & $520,42 * *$ \\
$(0,231)$ & $(0,138)$ & $(0,224)$ & $(0,001)$ & & \\
\hline
\end{tabular}

1 Número entre parênteses representa o erro-padrão.

$2 \mathrm{X}^{2}$ é o coeficiente de determinação múltiplo.

$*$ e ** Significativo a $5 \%$ e a $1 \%$ de probabilidade, respectivamente concentrado na dieta dos animais. Esta redução atingiu a cifra de $7 \%$ no nível $\mathrm{N}_{2}$ em relação ao nível $\mathrm{N}_{0}$ de suplementação e está em conformidade com os resultados obtidos por Barros et al. (1992). MorandFehr \& Sauvant (1980) e Ciciliano-Jones \& Murphy (1989) concluíram que o aumento do nível de concentrado na dieta de ruminantes resulta em incrementos na concentração de ácido propiônico e reduções na produção de ácido acético, no rúmem; segundo Kolb (1984), o ácido acético e o butírico participam com 60 a $80 \%$ na síntese de gordura do leite.

Os resultados da análise econômica apresentados na Tabela 6 mostram que, embora não tenha havido diferença $(p>0,05)$ significativa entre os níveis de suplementação utilizados, o nível $\mathrm{N}_{2}$ (300 g de concentrado/animal/dia) apresentou margem bruta com $8,3 \%$ e $9,2 \%$ de superioridade em relação aos níveis $\mathrm{N}_{0}$ e $\mathrm{N}_{1}$, respectivamente. Observando-se a Fig. 3, nota-se que a recuperação do peso corporal das matrizes foi mais rápida quando submetidas ao nível de suplementação $\mathrm{N}_{2}$ que nos demais níveis,

TABELA 5. Valores médios \pm erro-padrão de proteína bruta, sólidos totais e gordura do leite de cabras da raça Anglo-nubiana em pastoreio, submetidas a diferentes níveis de suplementação alimentar na região semi-árida.

\begin{tabular}{lrrr}
\hline \multirow{2}{*}{ Item } & \multicolumn{3}{c}{ Níveis de suplementação } \\
\cline { 2 - 4 } & \multicolumn{1}{c}{$\mathrm{N}_{0}$} & \multicolumn{1}{c}{$\mathrm{N}_{1}$} & \multicolumn{1}{c}{$\mathrm{N}_{2}$} \\
\hline Proteína bruta (\%) & $3,46+0,1$ & $3,37+0,03$ & $3,57+0,1$ \\
Sólidos totais (\%) & $13,09 \pm 0,2$ & $13,02 \pm 0,10$ & $12,87 \pm 0,3$ \\
Gordura (\%) & $3,85+0,1$ & $3,80+0,10$ & $3,59+0,1$ \\
\hline
\end{tabular}

TABELA 6. Estrutura de custos, receita e margem bruta da produção de leite, com diferentes níveis de suplementação de cabras da raça Anglo-nubiana, em pastoreio na região semi-árida.

\begin{tabular}{lrrr}
\hline Item & \multicolumn{3}{c}{ Níveis de suplementação } \\
\cline { 2 - 4 } & \multicolumn{1}{c}{$\mathrm{N}_{0}$} & \multicolumn{1}{c}{$\mathrm{N}_{1}$} & $\mathrm{~N}_{2}$ \\
\hline Receita (R/cab.) & 89,38 & 94,93 & 107,02 \\
$\quad$ Produção total de leite (kg/cabeça) & 111,73 & 118,66 & 133,77 \\
Custos variáveis (R\$/cabeça) & 5,92 & 12,31 & 16,54 \\
Concentrado & - & 4,23 & 8,46 \\
Forragem & 0,92 & 0,92 & 0,92 \\
Vacina (febre aftosa) & 0,35 & 0,35 & 0,35 \\
Vermifugação & 0,33 & 0,33 & 0,33 \\
Mão-de-obra & 4,32 & 6,48 & 6,48 \\
Margem bruta (R\$/cabeça) & 83,46 & 82,62 & 90,98 \\
\hline
\end{tabular}


aspecto importante para a próxima lactação. Assim, o $\mathrm{N}_{2}$ é a alternativa que poderá assegurar maior rentabilidade ao produtor.

\section{CONCLUSÕES}

1. A caatinga nativa raleada, na região de Sobral, suporta o nível de produção de leite de cabras Anglonubianas de $1,2 \mathrm{~kg} /$ dia.

2. A melhor opção para se produzir leite, em condições de caatinga raleada, com cabras Anglonubianas, durante a época chuvosa, é com suplementação de $300 \mathrm{~g}$ de concentrado/animal/dia.

\section{REFERÊNCIAS}

ASSOCIATION OF OFFICIAL AGRICULTURAL CHEMISTS (Washington, Estados Unidos). Official methods of analysis. Washington, 1975. $1024 \mathrm{p}$.

BAKER, G.A.; SOUZA NETO, J. de. Assessment of the potential of dual-purpose goats in Northeast Brazil. Small Ruminant Research, Amsterdam, v.2, p.97105,1989

BARROS, N.N.; MESQUITA, R.C.M.; SOUZA NETO, J.; ALVES, J.U.; BARBIERI, M.E. Efeito de níveis de energia sobre a produção de leite em cabras da raça Anglo-nubiana. Pesquisa Agropecuária Brasileira, Brasília, v.27, n.1, p.115-130, jan. 1992

CICILIANO-JONES, J.; MURPHY, M.R. Production of volatile fatty acids in the rumen and cecumcolon of steers as affected by forage: concentrate and forage physical form. Journal of Dairy Science, Champaign, v.72, n.2, p.485-492, 1989.

DEVENDRA, C. Potential of sheep and goats in less developed countries. Journal of Animal Science, Champaign, v.51, n.2, p.461-463, 1980.

FRENCH, M.H. Observations on the goat. Rome : FAO, 1970. 204p. (FAO. Agricultural Studies, 80).

GUTIERREZ, N.; DE BOER, J. Marketing and price formation for meat goats, hair sheep and their products in Ceara State, Northeast Brazil. In INTERNATIONAL CONFERENCE OF GOAT PRODUCTION AND DISEASE, 3., 1982, Tucson Proceedings. Scottsdale : Dairy Goat Journal Publishing Company, 1982. p.50-54

KIRMSE, R.D. Effects of clearcutting on forage production, quality and decomposition in the caatinga woodland of Northeast Brazil: implications to goat and sheep nutrition. Logan : Utah State University, 1984. 150p. Ph.D. Thesis.

KOLB, E. Fisiologia da absorção e da digestão. In: KOLB, E. Fisiologia veterinária. 4.ed. Rio de Janeiro : Koogan, 1984. p.105-207.

MORAND-FEHR, P.; SAUVANT, D. Composition and yield of goat milk as affected by nutritional manipulation. Journal of Dairy Science, Champaign, v.63, n.10, p.1671-1680, 1980.

NATIONAL RESEARCH COUNCIL. Subcommitee on Goat Nutrition. (Washington, Estados Unidos). Nutrients requeriments of goats. Washington : National Academic Press, 1981. 91p.

NEUMAIER, M.C. Peasant production in Northeast Brazil: the case of goat production in Cariris Velhos, Paraíba. Columbia, MO : University of Missouri, 1986. 72p. (Technical Report Series, 73).

PFISTER, J.A. Nutrition and feeding behavior of goat and sheep grazing deciduous shrubswoodland in Northeastern Brazil. Logan : Utah State University, 1983. 130p. Ph.D. Thesis.

QUARTERLY BULLETIN OF STATISTICS. Rome FAO, v.1, p.64, 1988.

QUARTERLY BULLETIN OF STATISTICS. Rome FAO, v.1, p.41, 1993.

RODRIGUES, A.; SOUZA, W.H.; BANDEIRA, D.A.; FIGUEIREDO, E.A.P. de. Avaliação de produção leiteira em caprinos da raça Anglo-nubiana, Pardo Alemã e Sem Raça Definida (SRD), no Estado da Paraíba. João Pessoa : EMEPA, 1992. 12p. (EMEPA. Boletim de Pesquisa, 5).

SANDS, M.; McDOWELL, R.E. The potential of the goat milk production in the tropics. Ithaca: Cornell University, 1978. 53p. (Cornell International Agriculture mimiograph, 60)

SANTOS, H.P.; FANCELLI, A.L.; ANDIA, L.H. Análise econômica de sistemas de rotação de culturas para trigo, num período de dois anos, sob sistema plantio direto. Pesquisa Agropecuária Brasileira, Brasília, v.32, n.11, p.1111-1117, nov. 1997.

SCHACHT, W.H.; KAWAS, J.R.; MALECHECK, J.C. Effect of supplemental urea and molasses on dry season weight gains of goats in semiarid tropical woodland, Brazil. Small Ruminant Research, Amsterdam, v.3, n.7, p.235-244, 1992

ZOMETA, C.A.; SHELTON, M.; SOUSA, W.H. de. Estudo comparativo de rações completas contendo uréia sobre a produção de leite de cabras em confinamento. João Pessoa : EMEPA, 1986. 7p. (EMEPA. Comunicado técnico, 26). 